\title{
Novel Access Control Mechanism Based New Chinese Remainder Theorem II (New Crt II)
}

\author{
Aremu Idris Aremu ${ }^{1}$, Ibitoye Akinfola Akinrinnola ${ }^{1}$, Nwaocha Vivian Ogochukwu ${ }^{2}$ \\ ${ }^{1}$ Computer Science Department, School of Technology, Lagos State Polytechnic, Ikorodu, Lagos, Nigeria \\ ${ }^{2}$ Computer Science Department, Faculty of Science, Nigeria Open University, Ikorodu, Lagos, Nigeria
}

\section{Email address:}

aremu.i@mylaspotech.edu.ng (A. I. Aremu), mideeanddee@hotmail.com (I. A. Akinrinnola)

\section{To cite this article:}

Aremu Idris Aremu, Ibitoye Akinfola Akinrinnola, Nwaocha Vivian Ogochukwu. Novel Access Control Mechanism Based New Chinese Remainder Theorem II (New Crt II).International Journal of Intelligent Information Systems. Vol. 10, No. 3, 2021, pp. 31-36.

doi: $10.11648 /$ j.ijiis. 20211003.12

Received: March 17, 2021; Accepted: March 29, 2021; Published: June 22, 2021

\begin{abstract}
Security is a very vital concern in information system in this modern day. The protection of confidential files and integrity of information kept in the database are also of great important, the security model play an important role in protecting the privacy and integrity of messages in the database from unlawful users is a formal method to verify and describe intricate information system. An access Control mechanism is a main strategy for prevention and protection of the classified files in a database; this is carried out by restricting rights of access for different approved users of these files. This paper proposes a novel access control mechanism based on Chinese remainder theorem II which implements a single-key-lock system to encrypt one key called a secret key which is used for both encryption and decryption in the electronic information system for accessing the database. The key to be used in the decryption process must be exchanged between the entities in communication using symmetric encryption for the users to have access to the database. This method represents flocks and keys which is highly efficient and proficient. Also, this implementation can be achieved using the Chinese remainder theorem which executes faster operations and enables simpler construction of keys and locks to be provide for user to have access to control.
\end{abstract}

Keywords: Access Control, Residue Number System (RNS), New Chinese Remainder Theorem II (New CRT II)

\section{Introduction}

Data can be allocated to different users in the database in a time sharing system at a different time. Thus, the major unease task is to maintain the content of the file in the database by the database system chic [1-3]. Information stored in the database need to be protected from being damage, change or distorted without been notice by the database chic. The system supervisor needs to guarantee each user of the database, the use of the database resources in conformity with the policies of the system administrators. Information newly generated can be distributed across an extensive network for many users in the database environment; the safety of independent data becomes a heightened concern [4]. Cloud computing allows data sharing amid distinctive users of the database, which can be achieved with various degree of receptive means. As a result, controlling access system and stout isolation would be required.
Information fortification can be accomplished by means of an access control method. Once demand is made to access a file, it is interrupted by the control system to check for the validation before access can be given to the user [5]. The emergence of multimedia knowledge and distributed systems, has introduced the sharing of digital information across distinct users in unambiguous system or institution. These include video duplicates or digital audio, personnel data, commercial specifications, and digital books, which is stored in a universal database. Digital files are tremendously useful and must be confidentially reserved; to safeguard the content of the files from illegal access is a focus of eminent attention in the discipline of information security. The security of files during information accessing, is determined by an access control system employed to permit or hinder the access rights of distinct authorized users, i.e., to determine the degree of access distinct users have to the classified digital files. Therefore, the intrinsic purpose of the access control system is to avoid an unapproved users from viewing modifying and 
damaging the digital files [6].

Therefore, an access control mechanism is a technology to secure the privileged files stored in a database by limiting the access rights of distinct authorized users of the files. [6].

An access control method is an assembly of components and methods that decide the order of admission by rightful users to activities determined by predefined access authorization and privileges defined in the access security strategy [7]. The elementary objective of this access control method is to regulate the user privileges and secure information from illegitimate access [8].

The residue number system (RNS) is a number system for representing integers which is capable of supporting parallel, high-speed arithmetic. This system also presents some valuable properties for error detection, error correction, and fault tolerance. It has several applications in computationintensive digital signal processing (DSP) operations, like Fast Fourier Transform, Discrete Fourier Transform, direct digital frequency synthesis, digital filtering, convolution, correlation etc [9].

Addition, subtraction, and multiplication, are arithmetic operations that can be performed independently and parallel in various residue channels more effectively than in the conventional binary systems. The adoption of RNS has offered notably effective improvements for distinct types of DSP applications [10].

\section{Data Conversion}

The forward conversion can be express as the execution of a forward converter which decomposes a weighted binary number into a residue represented number with regards to a moduli set, this implies the conversion from a conventional representation to a residue one by dividing the number $\mathrm{X}$ by each of the identified moduli and then collect their remainder [11]. For instance, given moduli set $\{3,4,5\}$, the number 4 can be represented in RNS as:

$$
x_{i}=|X|_{m_{i}} \ldots \ldots
$$

$$
x_{1}=|X|_{m_{1}}=|4|_{3}=1 ; x_{2}=|X|_{m_{2}}=|4|_{4}=0 ; x_{3}=|X|_{m_{3}}=|4|_{5}=4
$$

Thus, the RNS representation of 4 is $(1,0,4)_{R N S(3,4,5)}$.

The basic technique in converting binary to RNS was presented by $[11,12]$.

New CRTII: To determine the correct decimal representation of the RNS number $\mathrm{X}$ with residue representation $\left(x_{1}, x_{2}, \ldots x_{n}\right)$ with respect to $\left\{m_{1}, m_{2}, m_{3}, m_{4}\right\}$ is

$$
\mathrm{X}=x_{1}+m_{1}\left|k_{1}\left(x_{2}-x_{1}\right)+k_{2} m_{1}\left(x_{3}-x_{2}\right)+k_{3} m_{1} m_{2}\left(x_{4}-x_{3}\right)\right|_{m_{2} m_{3} m_{4}}
$$

The difference between CRT II and the CRT is noticeable. CRT II does not have big modulo operations as compare to CRT, New CRTII is bounded by size $\sqrt{M}$ [13].

Illustration:

Given that $\left\{m_{1}, m_{2}, m_{3}, m_{4}\right\}$ are set of relatively co-prime

$$
\mathrm{X}=x_{1}+m_{1}\left|k_{1}\left(x_{2}-x_{1}\right)+k_{2} m_{1}\left(x_{3}-x_{2}\right)+k_{3} m_{1} m_{2}\left(x_{4}-x_{3}\right)\right|_{m_{2} m_{3} m_{4}}
$$

such that

$$
\begin{aligned}
& \left|k_{1} m_{1}\right|_{m_{2} m_{3} m_{4}}=1 \\
& \left|k_{2} m_{1} m_{2}\right|_{m_{3} m_{4}}=1 \\
& \left|k_{3} m_{1} m_{2} m_{3}\right|_{m_{4}}=1
\end{aligned}
$$

Given the set of 4 moduli set of $m_{1}=2^{n}-1, m_{2}=2^{n}+$ $1, m_{3}=2^{2 n+1}-1$. By applying divide and conquer techniques we have two different sets of moduli i.e first moduli set $=\left\{m_{1}, m_{2}\right\}$ and second moduli set $=\left\{m_{3}, m_{4}\right\}$, we determine the decimal equivalent of first residue $\operatorname{set}\left\{x_{1}, x_{2}\right\}$ and second residue set $\left\{x_{3}, x_{4}\right\}$. New Chinese remainder theorem can be used to compute for $X_{12}$ and $X_{34}$ as follows.

$X_{12}=x_{1}+m_{1}\left|k_{1}\left(x_{2}-x_{1}\right)\right|_{m_{2}}=$ first residue w.r.t first moduli set

$X_{34}=x_{3}+m_{3}\left|k_{2}\left(x_{4}-x_{3}\right)\right|_{m_{4}}=$ second residue w.r.t second moduli set

By merging it together i.e. merge $\left\{X_{12}, X_{34}\right\}$ w.r.t number such that $\operatorname{gcd}\left(m_{1}, m_{2}\right)=1, \operatorname{gcd}\left(m_{1}, m_{3}\right)=1$, $\operatorname{gcd}\left(m_{1}, m_{4}\right)=1, \quad \operatorname{gcd}\left(m_{2}, m_{3}\right)=1, \quad \operatorname{gcd}\left(m_{2}, m_{4}\right)=1$, $\operatorname{gcd}\left(m_{3}, m_{4}\right)=1$. The decimal equivalent of residue representation of $\left\{x_{1}, x_{2}, x_{3}, x_{4}\right\}$ of an integer $\mathrm{X}$ is given as $\left\{m_{1}, m_{2}, m_{3}, m_{4}\right\}$

$$
\mathrm{X}=X_{12}+m_{1} m_{2}\left|k_{3}\left(X_{34}-X_{12}\right)\right|_{m_{3}, m_{4}}
$$

Demonstrating example:

$$
\begin{gathered}
\mathrm{X}=(1,2,3,4)_{R N S(3,5,7,11)} \\
X_{12}=x_{1}+m_{1}\left|k_{1}\left(x_{2}-x_{1}\right)\right|_{m_{2}} \\
x_{1}=1 m_{1}=3 m_{2}=5 \\
\left|k_{1} m_{1}\right|_{m_{2}}=1=\left|3^{-1}\right|_{5}=2 \\
1+3|2(2-1)|_{5}=\left.|| 2\right|_{5}+\left.|-1|_{5}\right|_{5} \\
1+3(2)=7 \\
X_{34}=x_{3}+m_{3}\left|k_{2}\left(x_{4}-x_{3}\right)\right|_{m_{4}} \\
x_{3}=3 m_{3}=7, m_{4}=11 x_{4}=4 \\
\left|k_{1} m_{3}\right|_{m_{4}}=1=\left|7^{-1}\right|_{11}=8
\end{gathered}
$$




$$
\begin{gathered}
X_{34}=3+7|8(4-3)|_{11} \\
3+7 * 8=59
\end{gathered}
$$

\section{Proposed Scheme}

Given a moduli set $\left\{m_{1}, m_{2}, m_{3}, m_{4}\right\}$, its equivalent weighted number $\mathrm{X}$ can be transformed from the residue representation $\left(x_{1}, x_{2}, x_{3}, x_{4}\right)$ by the definition of Divide and Conquer Techniques we have as follows:

$$
\begin{aligned}
& X_{12}=x_{1}+m_{1}\left|k_{1}\left(x_{2}-x_{1}\right)\right|_{m_{2}} \\
& X_{34}=x_{3}+m_{3}\left|k_{2}\left(x_{4}-x_{3}\right)\right|_{m_{4}}
\end{aligned}
$$

Such that

$$
\begin{gathered}
\left|k_{1} m_{1}\right|_{m_{2}}=1 \\
\left|k_{2} m_{3}\right|_{m_{4}}=1 \\
X=X_{12}+m_{1} m_{2}\left|k_{3}\left(X_{34}-X_{12}\right)\right|_{m_{3} m_{4}}
\end{gathered}
$$

And also,

$$
\left|k_{3} m_{1} m_{2}\right|_{m_{3} m_{4}}=1
$$

Theorem 1:

Given $\left\{2^{n}-1,2^{n}+1,2^{2 n}, 2^{2 n+1}-1\right\}$ moduli sets, by the definition of divide and conquer techniques, $m_{1}=2^{2 n+1}-1, m_{2}=2^{2 n}, m_{3}=2^{n}+1, m_{4}=2^{n}-1 \quad$ then, we have $\left\{m_{1}, m_{2}\right\}$ and $\left\{m_{3} m_{4}\right\}$ and by definition of $\left|k_{1} m_{1}\right|_{m_{2}}=1$ and $\left|k_{2} m_{3}\right|_{m_{4}}=1$ the following hold true:

$$
\begin{gathered}
k_{1}=1 \\
k_{2}=2^{n-1} \\
k_{3}=1
\end{gathered}
$$

Proof:

If it can be demonstrated that $2^{2 n+1}-1$ with respect to $2^{2 n}$ : $\left|2^{2 n+1}-1\right|_{2^{2 n}+1}=1$, then 1 is the multiplicative inverse of
$2^{2 n+1}-1$ with respect to $2^{2 n}$. Similarly, $2^{n}+1$ with respect to $2^{n}-1:\left|2^{n}+1 \times 2^{n-1}\right|_{2^{n}-1}=1$, then $2^{n-1}$ is the multiplicative inverse of $2^{n}+1$ with respect to $2^{n}-1$. And also, $2^{2 n-1}-1 \times 2^{2 n} \quad$ with respect to $2^{n}+1 \times 2^{n}-1$ : $\left|2^{2 n-1}-1 \times 2^{2 n} \times 1\right|_{2^{n}+1 \times 2^{n}-1}=1$, then 1 is the multiplicative inverse of $2^{2 n+1}-1 \times 2^{2 n}$ with respect to $2^{n}+1 \times 2^{n}-1$.

By the definition of $X_{12}=x_{1}+m_{1}\left|k_{1}\left(x_{2}-x_{1}\right)\right|_{m_{2}}$ and $X_{34}=x_{3}+m_{3}\left|k_{2}\left(x_{4}-x_{3}\right)\right|_{m_{4}}$ we have

$$
\begin{aligned}
& X_{12}=x_{1}+2^{2 n+1}-1\left|\left(x_{2}-x_{1}\right)\right|_{2^{2 n}} \\
& X_{34}=x_{3}+2^{n}+1\left|2^{n-1}\left(x_{4}-x^{3}\right)\right| 2^{n}-1
\end{aligned}
$$

And also by the definition of $X=X_{12}+m_{1} m_{2} \mid k_{3}\left(X_{34}-\right.$ $\left.X_{12}\right)\left.\right|_{m_{3} m_{4}}$ we have

$$
X=X_{12}+2^{2 n+1}-1 \times 2^{2 n}\left|X_{34}-X_{12}\right|_{2^{2 n}-1}
$$

Therefore the hardware realization of the proposed scheme will base on the following equations which can be further simplified as follow respectively:

$$
\begin{gathered}
X_{12}=x_{1}+m_{1}\left|\left(x_{2}-x_{1}\right)\right|_{2^{2 n}} \\
X_{34}=x_{3}+m_{3}\left|2^{n-1}\left(x_{4}-x^{3}\right)\right| 2^{n}-1 \\
X=X_{12}+2^{2 n+1}-1 \times 2^{2 n}\left|X_{34}-X_{12}\right|_{2^{2 n}-1} \\
X_{12}=A \\
X_{34}=B \\
X=C
\end{gathered}
$$

Where

$$
\begin{aligned}
& A=x_{1}+T \\
& B=x_{3}+U \\
& C=X_{34}+V
\end{aligned}
$$

$$
\begin{gathered}
x_{1}=\overbrace{x_{12 n-1} x_{12 n-2} x_{12 n-3} \ldots \ldots \ldots \ldots x_{11} x_{10}}^{2 n+1} \\
x_{2}=\overbrace{x_{22 n-1} x_{22 n-2} x_{22 n-3} \ldots \ldots \ldots \ldots x_{21} x_{20}}^{2 n}=\underbrace{00 \ldots \ldots 00}_{n} \overbrace{x_{3 n} x_{3 n-1} x_{3 n-2} \ldots \ldots \ldots \ldots x_{31} x_{30}}^{n+1} \\
x_{4}=\underbrace{00 \ldots \ldots .00}_{n} \overbrace{x_{4 n-1} x_{4 n-2} x_{4 n-3} \ldots \ldots \ldots \ldots . x_{41} x_{0}}^{n-1}
\end{gathered}
$$




$$
\begin{aligned}
& A=\overbrace{x_{12 n-1} x_{12 n-2} x_{12 n-3} \ldots \ldots \ldots \ldots x_{11} x_{10}}^{2 n+1}+\overbrace{11 \ldots \ldots 11}^{2 n+1}|\overbrace{x_{22 n-1} x_{22 n-2} x_{22 n-3} \cdots \ldots \ldots \ldots x_{21} x_{20}}^{2 n}+\overbrace{\bar{x}_{12 n-1} \bar{x}_{12 n-2} x_{12 n-3} \ldots \ldots \ldots \ldots x_{11} x_{10}}^{2 n+1}|_{2 n}^{2 n}
\end{aligned}
$$

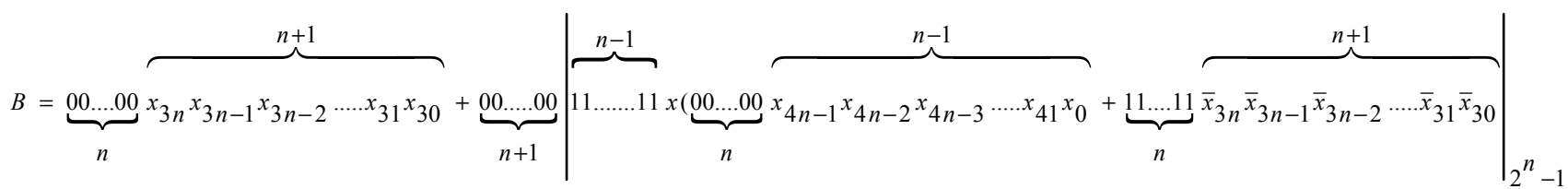

$$
\begin{aligned}
& x_{1}, x_{2}, x_{3} \text { and } x_{4} 2^{2 n+1}-1
\end{aligned}
$$

$$
C=X_{12}+\overbrace{11 \ldots \ldots \ldots 11}^{4 n+1}\left|X_{34}-X_{12}\right|_{2^{2 n}-1}
$$

We begin by explaining the component of the outcome from the New CRT II method, which is based on A, B and C. As it can be seen from equation $13 \mathrm{~A}, \mathrm{~B}$ and $\mathrm{C}$ are generated by Carry Save Adders (CSAs) with end around carries (EACs) taking the values $x_{1}, x_{2}, x_{3}$ and $x_{4}$. These values must be added modulo $2^{2 n+1}-1$ in order to derive A, i.e., with a one's complement adder, namely a Carry Propagate Adder (CPA) with EAC. Bits easily derived by concatenating the operand $x$ with the n-bit left shift of $A$. This concatenation does not require any additional hardware. The operands B required $2 \mathrm{n}$ bit which concatenation of $m_{3}$ which does not required any additional hardware also. And finally, $\mathrm{C}$ required $2 \mathrm{n}-1$ bits moduli adder and also concatenation last three and four moduli of the set.

Forward conversion in the $\left\{2^{n}-1,2^{n}+1,2^{2 n}, 2^{2 n+1}-1\right\}$ moduli sets is straightforward and simple logic circuits, involving moduli adders, will suffice for implementation. If we define, $m_{1}=2^{n}+1, m_{2}=2^{n}-1, m_{3}=2^{2 n}, m_{4}=2^{2 n+1}-1$, then any integer $X$ within the dynamic range $M=\left[0,2^{6 n+1}-2^{4 n+1}-2^{4 n}+2^{2 n}-1\right]$ where upper and of the range is $\left(m_{1} m_{2} m_{3} m_{4}\right)$ is uniquely defined by a residue set $\left(x_{1}, x_{2}, x_{3} x_{4}\right)$, where $x_{i}=|X|_{m_{i}}$ and $\mathrm{X}$ is $6 \mathrm{n}+1-$ bits:

$$
X=x_{42 n} x_{42 n-1} \ldots \ldots \ldots x_{2 n-1} x_{32 n-2} \ldots \ldots . . x_{2 n-2} x_{2} \ldots \ldots \ldots x_{1} x_{1} \ldots \ldots . x_{0}
$$

Residue are obtained by nominally dividing X by $m_{i}$, the residue $x_{3}$ is the easiest to compute. The $2 \mathrm{n}$ least significant bits constitute the remainder when $\mathrm{X}$ is divided by the least significant $2 \mathrm{n}$ bits of $\mathrm{X}$. these bits are obtained by nominally shifting to the right by $2 \mathrm{n}$ bits.

$$
\begin{aligned}
& X_{12}=x_{1}+m_{1}\left|\left(x_{2}-x_{1}\right)\right|_{2} 2 n \\
& X_{34}=x_{3}+m_{3}\left|2^{n-1}\left(x_{4}-x^{3}\right)\right| 2^{n}-1 \\
& X=X_{12}+2^{2 n+1}-1 \times 2^{2 n}\left|X_{34}-X_{12}\right| 2^{2 n}-1
\end{aligned}
$$

Normally, because the shift may be hardwired. In order to determine the residues $\left(x_{1}, x_{2}, x_{3} x_{4}\right)$ we first partition $\mathrm{X}$ into four 2 n-bit blocks $B_{1}, B_{2}, B_{3}$ and $B_{4}$.

$$
\begin{aligned}
& B_{1}=\sum_{j=4 n}^{6 n+1} x_{j} 2^{j-4 n} \\
& B_{2}=\sum_{j=2 n}^{4 n} x_{j} 2^{j-2 n} \\
& B_{3}=\sum_{j=n}^{2 n} x_{j} 2^{j-n} \\
& B_{3}=\sum_{j=0}^{n} x_{j} 2^{j}
\end{aligned}
$$

Then,

$$
X=B_{1} 2^{4 n}+B_{2} 2^{2 n}+B_{3}+B_{4}
$$

The residue $x_{1}$ is obtained as

$$
\begin{gathered}
x_{1}=|X|_{2^{n}+1}=\left|B_{1} 2^{4 n}+B_{2} 2^{2 n}+B_{3}+B_{4}\right|_{2^{n}+1} \\
x_{1}=\left|B_{1}+B_{2}+B_{3}+B_{4}\right|_{2^{n}+1} \\
x_{2}=|X|_{2^{n}-1}=\left|B_{1} 2^{4 n}+B_{2} 2^{2 n}+B_{3}+B_{4}\right|_{2^{n}-1} \\
x_{2}=\left|B_{1}+B_{2}+B_{3}+B_{4}\right|_{2^{n}-1} \\
x_{4}=|X|_{2^{2 n+1}-1}=\left|B_{1} 2^{4 n}+B_{2} 2^{2 n}+B_{3}+B_{4}\right|_{2^{2 n+1}-1} \\
x_{4}=\left|B_{1}+B_{2}+B_{3}+B_{4}\right|_{2^{2 n+1}-1}
\end{gathered}
$$




\section{An Application of New Chinese Remainder Theorem II to Access Control Method}

The information protection system is employed to secure the access right of the information saved in the computer [14].

Table 1. Access Control information.

\begin{tabular}{lllll}
\hline FILES & $\boldsymbol{F}_{\mathbf{1}}$ & $\boldsymbol{F}_{\mathbf{2}}$ & $\boldsymbol{F}_{\mathbf{3}}$ & $\mathbf{F 4}$ \\
\hline$U_{1}$ & 3 & 0 & 2 & 2 \\
$U_{2}$ & 2 & 0 & 2 & 0 \\
$U_{3}$ & 0 & 2 & 0 & 1 \\
$U_{4}$ & 4 & 1 & 1 & 2 \\
\hline
\end{tabular}

0: No access 1: Executing 2: Reading 3: Writing 4: Owning.

Table 1, above shows an access control with four user and files, where $U_{i}$ and $F_{i}$ are denoted as user $\mathrm{i}$ and $\mathrm{j}$, for users and files respectively.

First, the system assigns four relatively prime locks $L_{1}=10, L_{2}=17, L_{3}=21$ and $L_{4}=15$ to the files $F_{1}, F_{2}, F_{3}$ and $F_{4}$ respectively. These lock to control by the access rights for each file. Then, the system computes four keys by using forward conversion $K_{1}, K_{2}, K_{3}$ and $K_{4}$ by New CRT II for the four users $U_{1}, U_{2}, U_{3}$ and $U_{4}$, respectively.

\section{Performance Analysis}

This segment considers some issues about the proposed access control methods, as well asthe computational complexity related with the construction of the keys and the storage requirement for the keys and locks. The implementation of the proposed mechanism is based on below equation.

$$
\begin{aligned}
& X_{12}=x_{1}+m_{1}\left|\left(x_{2}-x_{1}\right)\right|_{2^{2 n}} \\
& X_{34}=x_{3}+m_{3}\left|2^{n-1}\left(x_{4}-x^{3}\right)\right| 2^{n}-1 \\
& X=X_{12}+2^{2 n+1}-1 \times 2^{2 n}\left|X_{34}-X_{12}\right|_{2^{2 n}-1}
\end{aligned}
$$

Assume that $\left\{m_{1} m_{2}, \ldots . m_{n}\right\}$ are set of moduli set of relatively co-prime integer called moduli sets, with residue representation of $\left\{x_{1}, x_{2}, \ldots \ldots x_{n}\right\}$ where the $\operatorname{GCD}\left(m_{i}, m_{j}\right)=1$. The New CRT II for moduli set that are relatively co-prime needs to compute the equation $X_{12}=x_{1}+m_{1}\left|\left(x_{2}-x_{1}\right)\right|_{2^{2 n}}$

$X_{34}=x_{3}+m_{3}\left|2^{n-1}\left(x_{4}-x^{3}\right)\right| 2^{n}-1$

such that $X=X_{12}+2^{2 n+1}-1 \times 2^{2 n}\left|X_{34}-X_{12}\right|_{2^{2 n}-1}$

$x_{12}=x_{1}+m_{1}\left|\left(x_{2}-x_{1}\right)\right|_{2} 2 n$ with constant time complexity i.e $0(1)$, $X_{34}=x_{3}+m_{3}\left|2^{n-1}\left(x_{4}-x^{3}\right)\right| 2^{n}-1$ with constant time complexity i.e. $0(1)$, which has to do with n-bit 1 's complement operation and $X=X_{12}+2^{2 n+1}-1 \times 2^{2 n}\left|X_{34}-X_{12}\right|_{2^{2 n}-1}$ it required $0(\operatorname{logn})$ and the hardware complexity are n-bit 1's complement operation and n-bit multiplication and n-bit 1's complement operation. The proposed scheme required $0(\log n)$ for the time complexity and five n-bit for the hardware (area) complexity.

Table 2. Hardware Computation Complexity comparison.

\begin{tabular}{llll}
\hline Mechanisms & Method & Time Complexity & Storage Requirement \\
\hline$[1]$ mechanism & GCRT & $0\left(n^{2} b^{2}\right)$ & $0(\mathrm{~m}+\mathrm{n})$ \\
{$[6]$ mechanism } & GART & $0\left(\mathrm{n} b^{2}\right)$ & $0(\mathrm{~m}+\mathrm{n})$ \\
Our proposed mechanism & CRT11 & $0(\log n)$ & $0(\operatorname{logm}+\mathrm{n})$ \\
\hline
\end{tabular}

So, $0(\log m+n)$ form keys and $n$ locks thus avoiding the overflow problem.

\section{Conclusion}

The efficient access control method proposed is predicated on the concept of the single key lock method, based on forward conversion and Chinese remainder theorem II (CRTII) for both key and lock respectively. The proposed method utilized an effective technique to produce keys for users. First, choosing n pairwise co-prime integers $L_{j}$ for $1 \leq \mathrm{j} \leq \mathrm{n}$ as the keys of the $\mathrm{n}$ files and determing the access right $a_{i j}$ of distinct user $U_{i}$ to each digital file $F_{j}$ in the invisible access control matrix. The key $K_{i}$ for distinct user $U_{i}$ can be generated easily by the locks, $L_{1}, L_{2}, \ldots \ldots L_{n}, U_{i}$ 's rights CRTII. We examined the time complexity of the CRTII and determined that the method are more efficient than [1].

\section{References}

[1] D. E. Derming and P. J. Denning, "Data Security," ACM Comp. Survey, Vol. 1 No. 3, (1979), pp 227-249.

[2] B. W. Lampson 'Protection" Proc. 5th Princeton Sytnp. of Info. Sci. and Syst., Princeton Univ., (1971), pp. 437-443.

[3] G. S. Graharn and P. L. Denning, "protection-principles and practice," Proc. Spring Jt. Computer Coference, Vol. 40, AFIPS Press, Montvale, N. J., (1972), pp. 417-29.

[4] Kim S. Lee et al. A Hierarchical Single-Key-Lock Access Control Using the Chinese Remainder Theorem. ACM 1992.

[5] Baumann, A., Peinado, M., \& Hunt, G. (2015). Shielding applications from an untrusted cloud with haven. ACM Transactions on Computer Systems (TOCS), 33 (3), 1-26.

[6] M. L. Wu, T. Y. Hwang, Access control with single-key-lock, IEEE Trans. Software Eng. 10 (2) (1994) 185-191. 
[7] Yanjun Liu, Chin-Chen, and Shih-Chang Chang, An Access Control Mechanism Based on the Generalized Aryabhata Remainder Theorem. International Journal of Network Security, Vol. 16, No. 1, PP. 58-64, Jan. 2014.

[8] Anderson R. Security Engineering: a guide to building dependable distributed systems. John Wiley \& Sons; 2010. p. 640 .

[9] Younis A. Younis, KashifKifayat, MadjidMerabti. An access control model for cloud computing. journal of information security and aplications 19 (2014) 45-60. Elsevier Ltd.

[10] Vilardy, J. M., Giacometto, F., Torres, C. O., \& Mattos, L. (2011, January). Design and implementation in VHDL code of the two-dimensional fast Fourier transform for frequency filtering, convolution and correlation operations. In Journal of Physics-Conference Series (Vol. 274, No. 1, p. 012048).

[11] Ouyang, J., Coatrieux, G., \& Shu, H. (2015). Robust hashing for image authentication using quaternion discrete Fourier transform and log-polar transform. Digital Signal Processing, 41, 98-109.

[12] Chervyakov, N. I., Molahosseini, A. S., Lyakhov, P. A., Babenko, M. G., \& Deryabin, M. A. (2017). Residue-tobinary conversion for general moduli sets based on approximate Chinese remainder theorem. International journal of computer mathematics, 94 (9), 1833-1849.

[13] C. H., Premkumar, A. B., \& Zhang, W. (2013). A new RNS based DA approach for inner product computation. IEEE Transactions on Circuits and Systems I: Regular Papers, 60 (8), 2139-2152. Omondi A. and Premkumar B (2007): Residue Number Systems: Theory and Implementation. Imperial College Press, 2007.

[14] Ouye, M. M., \& Crocker, S. T. (2018). U.S. Patent No. 10,033,700. Washington, DC: U.S. Patent and Trademark Office. 